\title{
Special Issue JOMS - Journal of Medical Systems, 2016 on Agent-Empowered HealthCare Systems
}

\author{
Pedro Henriques Abreu ${ }^{1}$ - Daniel Castro Silva ${ }^{2}$ - Michael Ignaz Schumacher ${ }^{3}$. \\ Luís Paulo Reis ${ }^{4}$ • Brígida Mónica Faria ${ }^{5}$ - Márcia Ito ${ }^{6}$
}

Published online: 10 February 2016

(C) Springer Science+Business Media New York 2016

\section{Preface}

The Agent-Empowered HealthCare Systems Special Issue includes a selection of papers that provide discussion on the most recent and innovative work regarding the study and application of agent-based technology to healthcare. Healthcare facilities faces now diverse and complex everyday challenges, ranging from equipment and drug inventory management to patient clinical record and follow-up management, or even patient monitoring in either a hospital or home environment, and patient transportation scheduling. At the same time, the progressive maturity of agent technology methodologies and tools has led to the development of applications in many complex real-world domains. Covering a wide spectrum of research areas, this special issue intends to

The Special Issue for this editorial can be found at the following link: http://link.springer.com/journal/10916/40/2/page/1

Pedro Henriques Abreu

pha@dei.uc.pt

Daniel Castro Silva

dcs@fe.up.pt

Michael Ignaz Schumacher

michael.schumacher@hevs.ch

Luís Paulo Reis

lpreis@dsi.uminho.pt

Brígida Mónica Faria

btf@estsp.ipp.pt

Márcia Ito

marciaito@br.ibm.com bring together communities from Computer Science and Agent-Based Systems and Clinicians working on Healthcare, Medicine and Biomedicine, providing a presentation and discussion forum for researchers in the area, highlighting innovative approaches, multidisciplinary works, and defining future research directions.

The special issue accepted submission of paper concerning the use of agents encompassed in the healthcare context - e.g. ranging from equipment and drug inventory management to patient clinical record and follow-up management, or even patient monitoring in either a hospital or home environment, and patient transportation scheduling. Topics of interest included: Medical Data Management, Information Retrieval and Knowledge Management, Agent-based Information Provider Services, Remote Care, and Telemedicine, Mobile

Faculty of Engineering, University of Porto, Rua Dr. Roberto Frias, s/n, 4200-465 Porto, Portugal

Institute of Business Information Systems, University of Applied Sciences Western Switzerland (HES-SO), Rue de la Jeunesse 1, 2800 Delémont, Switzerland

4 School of Engineering, University of Minho, Campus de Azurém, 4804-533 Guimarães, Portugal

5 Oporto Polytechnic Institute, Rua Dr. Roberto Frias, 4200-465 Porto, Portugal

6 Brazil IBM Research Lab, R. Tutóia, 1157, Vila Mariana, São Paulo, Brazil

\footnotetext{
Pinhal de Marrocos, 3030-290 Coimbra, Portugal
} 
Agents in Hospital Environments, - Robotics in Healthcare, Impact of Agents in Patient Quality of Life, Patient Empowerment through Personalized Agent-based Systems, Patient Management (e.g. through agent cooperation, distributed patient scheduling), Planning and Resource Allocation, Medical Agent-based Decision Support Systems, Agent-based Patient Monitoring and Diagnosis, Agent-based Medical Training and Education, Ethics/Legal Issues and Security in Healthcare Agent-based Systems.

As an answer to the call-for-papers for this special issue, a total of 18 papers with over 60 authors from 13 countries were submitted for evaluation. After a very rigorous review process, where each paper was reviewed by at least 3 reviewers, experts on the agent-empowered healthcare field or related fields, a total of 8 papers was accepted for publication in this Special Issue, corresponding to an acceptance rate of less than $45 \%$. These papers cover a broad range of active research areas in Agent-Empowered HealthCare Systems, including also a survey on agents applied in healthcare.

The special issue includes 8 contributions focusing distinct areas of Agent Empowered HealthCare Systems. The first paper deals with situated agents and humans in social interaction with application on elderly healthcare. It aims to develop a semi-autonomous, collaborative tool to promote monitored, intensive, extended and personalized therapeutic regime adherence at home based on adaptation. The second paper deals with the development of a mobile application for analyzing side effects of food additives via a concept based search mechanism. The third paper deals with medical information foraging and the use of cooperative reactive agents for information foraging. The next article discusses the creation of an agent-based simulation model for the introduction of care coordination capabilities into a complex system of care for patients with serious and persistent mental illness. The fifth paper answers to the need to support interdisciplinary healthcare teams by a multi-agent system that allows these teams to manage patients according to presentation-specific clinical workflows. The next work consists in a survey on the most recent literature (2009-2014) concerning intelligent agents applied in the healthcare domain. The paper also uses its main findings to provide a discussion of current trends and future research on this area. The seventh paper researches on the definition of programmable expert personal health systems to monitor patients affected by chronic diseases using agent oriented programming and mobile computing. Finally, the last paper focusses on the description of a multi-agent system, based on a collaborative agent interaction protocol, designed for integrated detection of pharmacovigilance signals.

The guest editors of this special edition for the Journal of Medical Systems are grateful both to the authors of all papers submitted and of the eight accepted papers, for their contributions and, to the reviewers, for their insightful and thorough reviews that enabled a very rigorous and complete review process. 